\title{
Towards a framework for creative online collaboration: A research on challenges and context
}

\author{
Björn Stockleben ${ }^{1} \cdot$ Martyn Thayne ${ }^{2}$. \\ Seija Jäminki ${ }^{3}$. Ilkka Haukijärvi ${ }^{4}$. \\ Nicholas Blessing Mavengere ${ }^{5}$. \\ Muhammet Demirbilek ${ }^{6} \cdot$ Mikko Ruohonen $^{5}$
}

Published online: 25 April 2016

(C) The Author(s) 2016. This article is published with open access at Springerlink.com

\begin{abstract}
The OnCreate project was initiated by ten universities with expertise in collaborative work in online-based learning environments and explores the specific challenges of implementing university courses in creative disciplines in such an environment. The first research phase comprises a literature search on creativity and
\end{abstract}

Martyn Thayne

mthayne@lincoln.ac.uk

Björn Stockleben

bjoern.stockleben@hs-magdeburg.de; bjoern.stockleben@gmail.com

Seija Jäminki

seija.jaminki@lapinamk.fi

Ilkka Haukijärvi

ilkka.haukijarvi@tamk.fi

Nicholas Blessing Mavengere

nicholas.mavengere@uta.fi

Muhammet Demirbilek

mdbilek@sdu.edu.tr

Mikko Ruohonen

mikko.j.ruohonen@uta.fi

1 University of Applied Sciences Magdeburg-Stendal, 39114 Magdeburg, Germany

2 School of Film and Media, University of Lincoln, Brayford Pool, Lincoln, UK

3 Lapland University of Applied Sciences, Jokiväylä 11 C, 96300 Rovaniemi, Finland

4 Tampere University of Applied Sciences, Kuntokatu 3, 33520 Tampere, Finland

5 University of Tampere, Kalevantie 4, 33014 Tampere, Finland

6 Süleyman Demirel University, 32260 Isparta, Turkey 
its contextual factors in online collaboration in an educational setting. From this research a first set of possible key challenges and contextual key factors has been selected and applied to categorize the results of interviews and surveys on current good practice in creative online collaboration among experienced online teachers from the partner universities. From the key findings we formulate hypotheses to guide future research towards a framework for creative online collaboration. At the same time, the results can serve as inspiration for the educational practice. Notable observations of the good practice research among the partner universities are that they realise innovative collaboration concepts usually on mashed-up environments of state-of-the-art web services rather than on the omnipresent learning management systems (such as Moodle or Blackboard). Also, they show a paradigm shift from teaching to coaching and promotion of an open peer-review culture among the students.

Keywords Online collaboration · Design processes · Creativity· eLearning · Digital work

\section{Introduction}

The opportunity for users to collaborate and explore different perspectives is an important element in the design of university courses, especially for participants who are learning or working at a distance (A. Herrington and Herrington 2006). Study programmes following blended-learning or even pure online approaches are becoming increasingly popular in higher education. Two main drivers for this development are increasing international cooperation among universities and with industry partners and the special requirements of students in continuing education programmes. Thus, the development of more robust frameworks, pedagogies and tools for collaboration across the Higher Education sector can help to produce more employable students who are better equipped to deal with the contemporary industrial setting (Okuogume and Jäminki 2011). According to research by the consultancy Woods Bagot (Holmes 2012), entitled University Graduates Don't Make the Grade and based on a survey of 500 business leaders, collaboration has become a key employment requirement that is currently being underserved in higher education. When asked, "what are the three most important skills or attributes new hires need in order to succeed at your organization" the top three were Problem Solving (49\%), Collaboration/Ability to Work as a Team (43\%) and Critical Thinking (36\%).

Hence, in September 2014, 10 universities from across Europe started an EU Erasmus + Strategic Partnership (Table 1). This joint project is about the exchange, implementation and evaluation of processual and contextual knowledge of online collaborative courses, with a focus on teaching creation and innovation processes. Apart from questions of choice of tools and platforms, the project will especially investigate how to create social presence and other soft contextual factors that foster creative collaboration in online learning environments. In physical rooms, we are used to creating spaces and processes to support ideation and creation processes (Doorley et al. 2011). The project seeks to create virtual spaces for creative learning in a similar manner, addressing the frequent problems we encounter in online collaboration. 
Table 1 OnCreate project consortium

\begin{tabular}{|c|c|c|}
\hline Partner & Related research \& teaching & Short \\
\hline $\begin{array}{l}\text { University of Applied Sciences } \\
\text { Magdeburg-Stendal, Germany }\end{array}$ & Master Cross Media ${ }^{a}$ blended learning programme & Magdeburg \\
\hline University of Lincoln, UK & Co_lab ${ }^{b}$ research group on creative collaboration & Lincoln \\
\hline $\begin{array}{l}\text { International YMCA University } \\
\text { Kassel, Germany }\end{array}$ & $\begin{array}{l}\text { Human Development online study programme } \\
\text { targeted at African students }\end{array}$ & Kassel \\
\hline $\begin{array}{l}\text { Süleyman Demirel University, } \\
\text { Isparta, Turkey }\end{array}$ & $\begin{array}{l}\text { Research activities on design and implementation of } \\
\text { educational technology and digital learning material. }\end{array}$ & Isparta \\
\hline $\begin{array}{l}\text { University of Applied Sciences } \\
\text { Potsdam, Germany }\end{array}$ & $\begin{array}{l}\text { Authors of the successful MOOC "The future of } \\
\text { storytelling"d with } 70.000+\text { participants }\end{array}$ & Potsdam \\
\hline University of Tampere, Finland & Global Virtual Collaboration Simulation VIBu ${ }^{\mathrm{e}}$ & Tampere \\
\hline $\begin{array}{l}\text { University of Applied Sciences } \\
\text { Tampere, Finland }\end{array}$ & $\begin{array}{l}\text { ProAcademy project-oriented learning, member of } \\
\text { European Virtual University }\end{array}$ & TAMK \\
\hline University of Aalborg, Denmark & $\begin{array}{l}\text { Expertise in project-based learning, } \mathrm{mICT}^{\mathrm{g}} \text { blended } \\
\text { learning master programme }\end{array}$ & Aalborg \\
\hline $\begin{array}{l}\text { University of Applied Sciences } \\
\text { Lapland, Finland }\end{array}$ & Online programmes in Visual Design and Fine Arts & Lapland \\
\hline University of Ljubljana, Slovenia & $\begin{array}{l}\text { Authors of E-CHO e-learning platform, production } \\
\text { of courses and materials }\end{array}$ & Ljubljana \\
\hline
\end{tabular}

\footnotetext{
${ }^{\mathrm{a}}$ http://www.ma-crossmedia.de

${ }^{\mathrm{b}}$ http://colab.lincoln.ac.uk/

${ }^{\mathrm{c}}$ http://www.cvjm-hochschule.de/study-programs/ba-in-human-development-hud/

${ }^{\mathrm{d}}$ https://iversity.org/en/courses/the-future-of-storytelling

e http://www.vibu.fi/

${ }^{\mathrm{f}} \mathrm{http}: / /$ www.evaonlinecourses.eu/

${ }^{\mathrm{g}} \mathrm{http}$ //www.en.aau.dk/education/continuing-education/master/information-and-communication-technologies

${ }^{\mathrm{h}} \mathrm{http} / /$ www.ltfe.org/category/english/projects/e-learning/
}

In a collaborative effort, the OnCreate project consortium agreed on the following working definition for its research matter of online collaborative creative processes:

"Online collaborative creative processes comprise all such activities which aim to
solve in a group problems that do not have standard solutions, mediated through
web-based tools. Typically, such problems require interdisciplinary, lateral think-
ing, social empathy and extensive ideation with the aim of mutual inspiration. The
processes applied are often nonlinear and rely on multimodal means of synchro-
nous and asynchronous communication, with a special focus on visual tools."

The applicability of this definition reaches out beyond the classical creative disciplines of design and storytelling on purpose. It builds on the principles of design thinking, as e.g. outlined by Brown (2008). For example, Lugmayr et al. (2013) describe how design thinking can be integrated into media management courses, which form an important part of the prospective OnCreate course portfolio. The working definition emphasizes that creative problem solving is a core skill applicable across a large number of disciplines to tackle complex problem-solving in modern societies. 


\section{Approach \& methodology}

The OnCreate project comprises the conception and evaluation of online-based courses in creative disciplines, as well as the creation of a generic guidance framework on the design of creativity-supporting online spaces. In an accompanying research strand, the consortium seeks to gather the theoretical foundations for such a framework. The research is divided into four consecutive activities, where this paper comprises results of the first and the second one:

1. Literature review on the foundations of creativity, online collaboration and learning models

2. Assessment of good practice examples of creative online collaboration among the participating universities

3. Assessment of good practice examples of creative online collaboration outside the consortium, especially in enterprises

4. Experiments on particular aspects of creative online collaboration

From the findings of the literature review, two category sets were derived. One contains challenges of collaborative creative processes as such (Table 2), the other addresses contextual factors for creative online learning spaces (Table 3). These serve as an open draft framework, intended to be altered and extended by the findings of the subsequent research activities.

The OnCreate consortium constitutes an implicit qualitative pre-selection of interview partners in the sense that the consortium was selected according to the criteria of notable prior experiences and research activities related to online collaboration. While it would be pretentious to claim they were a representative sample of the innovators in this area, it is safe to say that they have a progressive and embracing attitude towards the topic. The assessment of good practice in creative online collaboration among the

Table 2 Challenges of creative processes in online environments

\begin{tabular}{ll} 
Characteristics of creative processes & Challenge in online environments \\
\hline $\begin{array}{l}\text { Mutually shared understanding of } \\
\text { viewpoints (empathy) and knowledge }\end{array}$ & $\begin{array}{c}\text { Online environments fall short of emotional cues and informal } \\
\text { conversations. Substitutes for both are needed to build a } \\
\text { successful team. } \\
\text { Unique tasks }\end{array}$ \\
$\begin{array}{l}\text { Creative processes know a plethora of methods to tackle any } \\
\text { creative challenge, but only a handful of them has been } \\
\text { implemented online yet. }\end{array}$ \\
$\begin{array}{c}\text { The flexibility of online-supported processes has to be increased } \\
\text { to adapt processes quickly. }\end{array}$ \\
$\begin{array}{c}\text { Creative works are judged by a lot of different criteria and } \\
\text { decision-making is complex. Solutions to deeply discuss and } \\
\text { decide about creative work online are needed. }\end{array}$ \\
$\begin{array}{c}\text { Especially synchronous online methods are centred on a leader } \\
\text { or moderator. Online creative processes should have the } \\
\text { possibility to make anyone an actor driving the process at } \\
\text { any time. }\end{array}$
\end{tabular}


Table 3 Draft criteria and guiding research questions for instructor-centred aspects of environments for creative collaborative learning

Contextual factor

Facilitation and instructor feedback

Reflection of the collaboration processes

Access to facilitation

Instructor feedback

Technology environment and tools support Perceived user experience

Alignment between model of learning and communication model of learning platform

Emotional cues

Interoperability with different devices

Platform support
Guiding questions

How does facilitation include the reflection of the collaboration processes?

When and through which channels is the facilitator / Instructor available?

How and through which channels does the instructor give feedback on creative artifacts?

Do students feel confident they can solve the tasks given with the tools provided? Do they enjoy using the tools? Is this evaluated and if so, how?

Which criteria are applied when choosing tools for online learning?

Which role does non-verbal communication play in the course setting?

How far is ubiquitous access with different devices supported?

How do students get support in case of blocking technical problems? How are teachers supported to working with the platform?

participating universities was done in two steps. First the consortium gathered descriptions of already implemented courses employing creative online collaboration. For this, an online survey was created, where course descriptions along with the online collaboration approach were described by the partners in free text, yielding 18 entries. The second step had the form of five partner interview sessions, where two representatives from two universities compiled their experiences with creative online collaboration in a 90 min' session, with alternating the roles of interviewer and interviewee. The findings were captured on Google Docs during the interview and edited (i.e. enriched with more explanations and contextual information) by both partners after the session. The selected representatives fulfilled two criteria: They are experienced in online-based teaching and they are involved in their schools' research and innovation activities in online-learning. In this respect, they can be considered experts in the field of online learning and collaboration. The form of an open partner interview was chosen, as both the intrinsic curiosity and the comparison of similarities and differences in the approaches to creative online learning were deemed useful to trigger extensive experience reports, covering a wide range of aspects.

Both the survey and the interview instructions informed the participants to focus on good practice examples. We consider methods and didactic concepts as good practice if they have been applied to the subjective satisfaction of staff and students in a university and are going to be applied in future courses. Participants were encouraged to look at both successful established courses and promising recent pilots. In the interview, they were instructed to mutually ask questions as if they intended to implement the 
respective good practice examples in their own contexts. At interview time the literature-based draft framework was not disclosed to the interview partners.

Both the survey responses and the finished reports were then coded along the two category sets of challenges in the creative process (Table 2) and contextual factors (Table 3) for creative online learning spaces. Text passages of the report, usually of a length of one or two sentences, were coded along the pre-defined criteria. If a passage matched two criteria, it was coded for both. Text passages that did not fit any of the criteria, but with relevance to the theme of creative online collaboration were collected in an open category. The reason to applying a preliminary framework drawn from literature was to identify both new impulses from the actual teaching practice and lack of application of theory. Ideally, content that does not fit the framework indicates possible new areas of research, while empty or sparsely filled categories might indicate the need for a better theory-practice transfer or practical irrelevance of a category. The results were then summarized per category with two aims in mind: First, to get a summarized overview of the state of online collaboration at the participating universities as a basis for the conception of new courses in OnCreate. Second, to discuss the draft framework and generate first hypotheses for follow-up research.

\subsection{Limitations of the approach}

The chosen approach is limited in a number of ways. Most important, the sample of 10 universities is too small to generalize any findings beyond the scope of the project consortium itself. The coding categories were derived from multi-disciplinary literature sources and cannot be argued to be exhaustive. The purpose of this initial research activity was to harvest implicit knowledge from online collaboration innovators at the participating universities, document them in a structured way and derive hypotheses for future research in the project. However, the chosen approach does not allow interpretations on correlations between the different categories or their relative influence on the overall quality of creative online collaboration.

\section{Creative collaboration in the context of learning theories}

A theoretical basis for collaborative learning was provided by Johnson and Johnson (1996) in terms of cognitive development theories and especially from a Vygotskian perspective; on the basis of social independence theory. According to Vygotsky (1978), this type of social interaction involving cooperative or collaborative dialogue promotes cognitive development. Collaborative learning thus is a critical component that helps ensure quality in associated teaching and learning processes. The greater a student's involvement in the design, assessment and evaluation of their learning, the greater their motivation and the stronger the sense of responsibility for their own learning process is (Holmes 2012).

Such research demonstrates a necessity to understand more about the tools and frameworks needed to facilitate cooperative student partnerships and collaborative learning. Leidner and Jarvenpaa (1995) suggested that the effectiveness of information technology in contributing to learning will be a function of how well the technology supports a particular model of learning and the appropriateness of the model to a 
particular learning situation. Out of the learning models they mention in their paper, they mention three models with particular relevance for creative collaboration in online environments: Creativity goes along well with the principles of constructivism, as the process of design can be seen as a process of recombination of formerly internalized experiences into something novel. As Leidner and Jarvenpaa (1995) summarize, the constructivist model of learning assumes that individuals learn better when they are encouraged to explore a topic themselves. Collaborativism builds on this model, but emphasizes the importance of communication and creation of shared understandings in peer groups. This suits the creative process well, as it typically includes externalization and discussion of ideas, as well as inspiration from everyday experiences. This process has been well described by Buxton (2010) in his book Sketching User Experiences. Leidner and Jarvenpaa (1995) also mention Socioculturalism as a related learning model that emphasizes the importance of the learner's cultural and historical background for the construction of knowledge and the need to accommodate multiple viewpoints and opinions in teaching. This approach seems especially suitable for teaching design processes, where the quality of a solution is never absolute, but dependent on a plethora of contextual factors. Design does not know a final truth, only more or less viable solutions to a problem.

\section{Characteristics of creative collaboration}

Creativity is defined as an ability to produce a product or idea that is either original or possessing a new quality which people have not experienced before (Runco and Jaeger 2012). Collaboration is defined as an action or interpersonal relationship between professionals to achieve a specific purpose (Schrage 1995). It is a methodology through which work is produced by more than one person. In order to contribute effectively, collaborating partners should engage in a continuous reflective discourse on their work (Schoenfeld 1999). Creative collaborators are people who are interacting with others to discover genuinely new ways of thinking and doing something new together. Collaborators play principal roles by conceiving and carrying out the work.

Creativity used to be known as an individual characteristic, yet today it is increasingly seen as a shared and collaborative process. Therefore, the concept of creativity can be distinguished into individual and collaborative creativity. According to Romero and Barberà (2014) the latter is supposed to emerge in group settings depending on 16 contextual parameters compiled in the Assessment Scale for Creative Collaboration. They emphasize that creativity is not only a single idea or act but also the whole process of creating new and innovative solutions collaboratively in a group.

Creative collaboration is a process of exchanging of ideas among the participants helping to stimulate and enrich their own creativity to the extent that the solution they reach is novel and appropriate (Hong 2013). The characteristics of good creative collaboration are unique tasks, unpredictable results, shared understanding, communication, and joint decision-making (Kalay 2006). It requires higher cognitive taxonomy and critical reflections on other collaborative team members' social and educational background. It allows each team member to criticize ideas and make new propositions, which are iteratively integrated into the emerging collective creation. The purpose of collaboration rests on the limits of individual abilities which prevent completing a 
given task and the expectation that collaboration can help people complete the task more quickly and more effectively as well as produce better quality than they could otherwise (Kalay 2006).

The creative potential of online collaboration emerges in many forms, from epic cultural efforts such as Wikipedia, to open-source software, to smaller communities. Collaborative work can improve outcomes for both the individual and the community as a whole. According to Slavin (1980), working with others to achieve shared goals can promote social, motivational, and emotional benefits. For instance, collaboration in classroom settings can improve peer relations, increase self-esteem and develop perspective taking skills. Burke and Settles (2011) found that online creative collaboration processes improved the pursuit of the peers' own goals while at the same time made them behave in more community-favourable ways. Factors that affect the quality of collaboration are communication between collaborators, collaborations formed out of shared interests but offering different skills, and a similar level of skills and experience among the collaboration partners (Dow and Settles 2013).

According to Kim et al. (2014) asymmetric creative contributions may support a broad new class of creative collaborations. Asymmetric creative collaboration assigns different roles to the collaborators: One or more editors coordinate the creative process and assign specific tasks to contributors, which is essentially an application of the crowdsourcing approach (Estellés-Arolas and González-Ladrón-de-Guevara 2012) in the creative domain. Lahti et al. (2004) investigated the effectiveness of a computer supported collaborative environment, which allows participants to post their sketches and images remotely, for sharing and building knowledge amongst collaborators. They asked teachers, undergraduate students, and clients to use computer supported collaborative tools for sharing knowledge and ideas. They found that a networked collaborative learning environment facilitates students' engagement in sharing design problems, knowledge, and collaborators' ideas. Further studies of online collaboration have noted that online collaboration has positively contributed to student achievement and gratification of the collaborative environment (Godwin-Jones 2003; Haythornthwaite 2006).

Derived from the initial literature review above, Table 2 maps the characteristics of the creative process as described by Kalay (2006) with possible challenges in online environments. This will serve as an initial framework for the content analysis of the good practice research inside the consortium with focus on the implementation on the creative process.

The focus of the project is on online-based collaborative creative processes. Usually these processes are centred around very concrete problems, often implemented as project-based learning. Such kinds of courses have a high realism of context and the learning environment is in control of the group of learners. Knowledge is personally experienced and translated into competencies by the authentic learning context given (J. Herrington and Parker 2013). More particularly, in project-based learning prior knowledge is shared among the peer group and integrated into competencies by students.

\section{Contextual factors for the design of creative online learning spaces}

While the section before dwelled on the notion of creativity as such, this section describes a number of contextual factors in control of the teacher or facilitator who sets 
up and runs a course or project using creative online collaboration. It should be noted that the choice of factors can neither be exhaustive nor verified at this time, instead they form part of a framework for evaluation of creative online collaboration courses that is developed iteratively during the lifetime of the OnCreate project. At the end of this section, Table 3 summarizes the findings as a number of key contextual factors.

\subsection{Facilitation and instructor feedback}

Design and facilitation of the creative processes - the students' work - and the design of the learning environment are essential in terms of establishing an effective learner community. The instructor's presence has an effect on student satisfaction, sense of community and emotional connectedness, through design and facilitation of the social and cognitive processes (Jäminki 2008; Shea et al. 2005; Swan et al. 2009). Swan and Shih (2005) noted that also the course design affects the establishment of sense of community and emotional connectedness.

Since both the cognitive and social processes take place in online settings, an essential part of high quality facilitation is the availability of the instructor and the clarity and accuracy of the feedback given to and interaction with the students. Richardson and Swan (2003) and Swan et al. (2000) noted that student-teacher interaction has a correlation with learning outcomes. Instructor availability in these settings refers to being able to contact the instructor through different communication channels, easily and in timely manner (Brindley et al. 2009). A special challenge is commenting on visual artifacts, such as drafts and collections of material. In a physical setting, the instructor may simply point to and comment on particular aspects of a draft. In an online setting, this is limited depending on the channel used for giving feedback: The closest substitute for the index finger is the mouse pointer in an online meeting using screen sharing, the farthest situation is a comment in written text, where the location and quality of a visual artifact have first to be described in words before the actual comment may follow.

\subsection{Technology environment and tools support}

Designing a usable and user (student) friendly technological setting for the creative processes to take place in, requires holistic user-centric design thinking. As in any system or application use, perceived usability and usefulness of the tool have a direct impact on the actual use and user experience (student experience) (Bagozzi et al. 1992; Davis et al. 1989). From the pedagogic perspective, the effectiveness of an information system is defined by how well it aligns with the chosen model of learning and how well the model itself is aligned with the particular learning situation (Leidner and Jarvenpaa 1995). Also, since online settings fall short of traditional emotional cues and informal conversations, substitutes for these are required within the chosen tools to be used (Thayne et al. 2015).

Brindley et al. (2009) write that the accessibility of the environment must not be dependent on the devices the students use. Given the safe assumption that the personal technological environment of the student population can be diverse, this issue is a design challenge to be taken seriously, both, from institutional and individual perspectives. The fact that technological independency improves accessibility, acts as a strong relevance 
factor for higher education institutions, which are investing in and progressing with the so called Bring your own device (BYOD) culture and related practices.

On many occasions, the educational institution - or its suppliers - provides the technological support for the technologies used. Aside from facilitating the social and cognitive processes, on some occasions, also the instructor may be required to take the role of technical support as well. In online settings, the role of the technical support is critical, and in worst cases, inadequate support may prevent the creative processes from taking place. Thus, adequate and easily accessible support must be provided (Selim 2007). This is especially true for the field of lifelong learning, where students do have very rigid personal schedules without the option to shift work to the next day when the problem has been solved.

From the technological perspective, a crucial part of modern day's educational designer's - such as a teacher - competencies are the information system design capabilities to establish an effective and high quality collaborative learning environment, and willingness to constantly build up awareness of the user (student) expectations and developments in the field educational technology.

\section{Summary of good practice research}

This section summarizes the findings of the good practice research among the participating universities along the characteristics of the creative process and the contextual factors outlined in the prior literature search. If an example or experience can be attributed to a particular partner, the partner is mentioned explicitly. Whilst the tools and frameworks currently being used to facilitate collaboration vary between each partner, the collection of good practices from across the partnership has identified a number of common structural issues, technical requirements and relevant pedagogies for facilitating collaboration. These issues are discussed below and will be evaluated and explored further throughout the lifetime of the OnCreate project.

\subsection{Creative process}

\subsubsection{Mutually shared understanding of viewpoints (empathy) and knowledge}

A large number of partners are using different kinds of peer review methods to strengthen empathy and knowledge exchange in creative project work. A number of different modes of implementation is mentioned. At Aalborg, for example, student teams mutually act as consultants, reviewing and commenting on different stages of a project. The design management programme at TAMK uses open blogs, so even the project progress is visible continuously to other teams. Another effect of having a public blog is that the team has a common display of the state of the project, which can be discussed with teammates and external partners. This supposedly will lead to a better common understanding of project goals and ideas. The same open principles were applied in a recent online course on scriptwriting, where all scripts were open to all course members and had open discussion threads around them for everybody to participate.

While blogs usually allow for free-form articles well suited to communicate creative standpoints, Isparta uses tools available on the open source LMS Moodle to structure 
and build up collective knowledge. A glossary component is useful to establish a shared vocabulary in heterogeneous teams, an insight that is congruent with Magdeburg's experience in interdisciplinary online teamwork. For building more complex knowledge structures, the wiki feature is used, along with asynchronous threaded discussions.

At Magdeburg, student project teams agree on an initial set of tools for online collaboration. Basecamp ${ }^{1}$ and Adobe Connect are employed for asynchronous and synchronous communication respectively. As the project evolves the team usually enhances the toolset with further collaboration tools suited to special problems, e.g. mind-mapping services and online-pinboards such as Realtimeboard ${ }^{2}$ or Padlet ${ }^{3}$ for visual exchange of ideas. For collaborative text documents, Google Docs appears to be the de facto standard. At Lincoln, the teams developed an own style of commenting and marking collaborative documents, using colour codes. This is, as a side remark, a recurring observation, that tool deficits are alleviated by workarounds manifested in mutual conventions between the users.

\subsubsection{Unique tasks}

In general, the fact that creative projects usually deal with unique tasks seems to be no particular problem in online collaboration for the OnCreate partners, as no directly matching issues were found for this category during text analysis. Another interpretation might be that before specific methods and tools are developed, a stable basic set of generic tools has to be established and this might be the stage the partners are currently in. However, there still were a few references that relate to the idea of unique tasks in creative processes.

Lincoln made good experiences on pitching ideas for course material on Facebook in order to prepare workshops that better suit the actual needs of the students' concepts. Only if the proposed material got a minimum of 10 likes on Facebook, the teachers would include it into the workshop. This method encouraged student engagement with the design of the course and helped tailor the methods taught to the actual creative problems at hand. This was continued throughout the workshop phase, where both student and lecturers would suggest trips, cultural events, exhibitions and screenings which would only go ahead if there were enough likes.

Magdeburg often uses chosen methods from Design Thinking frameworks such as the human-centered design toolkit as provided by the design consultancy IDEO. These methods often employ specific ways for visualization. Sometimes they do include building physical prototypes, sometimes they have the need to collect photos or other media. Communicating and discussing the results of such methods is a challenge that is usually met by introducing generic online pinboard tools to the project collaboration environment, while very specific parts of the methods are still executed physically. As an example, prototypes are often still built with cardboard paper and then photographed and shared in a PDF or on an online pinboard, instead of using dedicated collaborative prototyping tools. This might be, however, characteristic to a transitional phase in digital work.

\footnotetext{
${ }^{1} \mathrm{http}: / /$ basecamp.com

$2 \mathrm{http}: / /$ realtimeboard.com

${ }^{3} \mathrm{http}: / /$ padlet.com
} 
Experiences at TAMK Proacademy and Floworks Living Lab indicate that students are usually more capable of designing their own learning and project environments and processes when they are mentored by experienced coaches.

\subsubsection{Unpredictable results}

The challenges of unique tasks and unpredictable results are related logically. The more open a creative process is, the less can the expected outcome be specified. The creative projects at the participating universities can be classified into two different types. For the first type, the outcome has a defined form, but open content. This applies mostly to projects in the area of visual design and media content, like branding design, videos or script, but also areas of management such as business plan development. These creative tasks follow well-defined processes with distinct tools. The second type are projects in the domain of application and product design and development, which either define a very generic form (such as an app) or even no fixed form at all, where the problem is the only determinant for the character of the solution. Typically, an important part of the second type of project is identifying an actually solvable problem within a larger problem space described in the briefing. Experiences with such projects in Aalborg and Magdeburg show that finding the right problem often takes a couple of weeks and is crucial for overall project success. This second type of project has a much more open design process and the choice of suitable methods and tools has a higher impact on the project outcome.

One of the challenges in planning a design process when the nature and quality of results is unpredictable is determining whether a result (i.e. an idea, concept or prototype) provides a viable solution to the formulated problem space. Especially Lapland mentioned the importance of authentic learning contexts, i.e. the project work should be based on authentic problems from the real world, as it is the case with industry cooperation for example. If exercises are simply constructed academically, they do not offer the depths necessary to verify the suitability of creative results. Apart from this, working on authentic problems is a high motivation factor for students, as observed by Magdeburg. Both universities let students work in cross-disciplinary groups to develop innovative ideas for enterprises. Lapland and TAMK mention ways to award the best ideas to add additional incentives.

\subsubsection{Joint decision-making}

An interesting model of joint decision making has already been mentioned earlier, which is the pitching of course content on Facebook as depicted by Lincoln. Aalborg gives initial rules for collaboration and encourages monthly feedback sessions on the teamwork as such. Magdeburg has a similar approach, by requiring two mandatory feedback sessions on team collaboration in a project. Also, Magdeburg emphasizes the importance of creative decisions through consent, not by majority vote. Their experience is that decisions through consent extend the duration of the ideation process, but yield better results and a higher general team motivation, which is critical for online collaborative projects where it is easy to simply hide away from the discourse. The $\mathrm{ViBu}^{4}$ sessions on global online teamwork in business management as frequently

${ }^{4}$ http://www.vibu.fi/ 
arranged by University of Tampere show an extreme example of collaborative online decisions under time pressure. Lapland includes exercises on negotiation in their online courses.

\subsubsection{Small status differences / flat hierarchies}

Among the participating universities, teaching in the creative domain develops strongly towards coaching and project oriented teaching among the project partners. While Aalborg University has long been known for its unparalleled project focus in teaching (15 out of 30 credit points of a semester is dedicated to collaborative project work), other partners have started initiatives on this in recent years.

The widely used learning management system Moodle, which is also deployed at the majority of the OnCreate partner universities, has a very strict, hierarchical and teacher-centred role scheme. For this reason, Magdeburg tends to use external web services for creative collaboration and also other partners prefer tools that support flat team hierarchies by design. Lincoln reported from their Media Culture

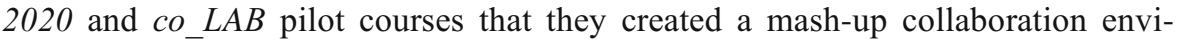
ronment comprised of Google Drive and Google Apps for content creation and Facebook as an informal coffee room area. In this shared working space, no one acts as administrator or gatekeeper, the teachers act as peers in the course. That said, it was also noticed that students often form their own working groups on Facebook, closed off from staff most of the time. This raises the question about how far students are ready to accept flat hierarchies or where they still expect to be led by a teacher.

At Potsdam, a dedicated online learning management system for the design disciplines called incom.org is developed and maintained. Apart from a strong focus on usability and visual design, it completely abandoned the idea of implementing different rights models for teachers and students directly on platform level. Instead, the roles on the platform are negotiated continuously and with respect to the course or project context at hand. The system is optimized for presentation and commenting of visual artefacts and can also be used as an online-portfolio of their study records for purposes like job applications. A number of dedicated content types is available, recently a template for scientific papers was added.

\subsection{Context of creative online learning spaces}

\subsubsection{Reflection of the collaboration process}

As in project work the actual working process is largely invisible to the supervisors, Magdeburg uses ePortfolios and project diaries for a continuous documentation and reflection of design processes and the overall learning progress. Especially project diaries, when written consecutively, give a good insight into the basics of creative decisions. Similar approaches are followed by Kassel with reflective essays and open blogs in the design management course at TAMK. Isparta encourages students in certain modules to chart their progress on blogs. A university-wide installation of wordpress is available for free to the students, but some use external platforms such as blogger.com or tumblr.com. 


\subsubsection{Access to facilitation}

For online course facilitation a number of partners emphasize the use of asynchronous forums on Moodle, assuring at least that questions can be asked at any time, although questions posted in the evening are usually answered the next working day. For the human development study programme at Kassel, asynchronous forums are the main way to communicate, as synchronous collaboration channels are usually too unstable in intercontinental connections to Africa, especially when distributed groups are connected. Aalborg reported similar findings from their $m I C T$ programme with participants from West Africa, though their use of tools was more diverse and included video conferencing.

An interesting special case of synchronous online facilitation is mentioned by Magdeburg: In a user experience research session, the panel members were grouped in pairs and assigned to separate breakout rooms in Adobe Connect. The task was to create user stories for a certain online service. They got a pre-structured form sheet on Google Docs to work on. The facilitator was jumping from room to room every 13 min to observe the progress, answer questions and give hints and inspiration. The combined creative output in this session was decisively larger and appeared to be of higher quality than could be expected from a doing the exercise in plenary. The transition between the rooms was relatively smooth for the facilitator and did not interfere with the ongoing creative process.

Lapland emphasized that the teaching staff have to be able to work and teach in teams, understand the principles of group dynamics, be competent with the use of online tools and understand the pedagogical implications of the tools applied.

\subsubsection{Instructor feedback}

This factor of instructor feedback is related to the demand for flat hierarchies in creative processes, because feedback can be given in a different tone, such as authorative, supporting or inspiring, determining the hierarchy between the student and the instructor. As already described, a lot of partners see the facilitation of a peer feedback culture and self-assessment as vital elements for teaching creative disciplines. An open question is, to which extent the former teacher role should turn into a pure coaching role, where the acquisition of new skills is purely student-driven. TAMK Proacademy is a notable pilot of such a purist approach, where the students found and lead own enterprises at the start of the study programme and meet in weekly workshops to exchange problems, research into literature to solve them jointly and hold presentations for the other peers. The role of the teacher is mainly to facilitate this process, not to drive a curriculum. In winter 2014, students and teachers from Lincoln, Tampere University and Magdeburg joined the IDEO MOOC on human-centered design, so teachers would experience the same challenges as the students and the course was effectively a co-learning situation rather than a teacher-student relation.

\subsubsection{Perceived user experience}

A prevailing impression among the interviewees is that the all-purpose learning management systems (LMS) such as Blackboard and Moodle deployed at most 
universities are rarely used for pilot projects on innovative forms of teaching. Lincoln mentions, that although Blackboard is the institutional platform for hosting all course documents, lecture slides, announcements and assignment submissions, no one really likes using it, neither staff nor students. It is said to be slow and offline a lot. Magdeburg complains that despite the variety of tools available in Moodle most of them are not really useful, as they are by far inferior to specialized solutions (e.g. for wikis, chats). For design faculties, the expectations of the look and feel (an important sub-aspect of the overall user experience) of state of the art web services are not fulfilled by widespread LMS, which especially Potsdam mentions as one of the motivations to develop the aforementioned platform incom.org.

\subsubsection{Alignment between model of learning and communication model of learning platform}

Any platform used for creative online collaboration has to support a collaborative communication model at its core. Offering a number of different communication tools from messaging over chat to wiki, it might seem an easy requirement to fulfil for any learning management system. Yet taking a closer look, the standard platforms deployed at the participating universities show serious shortcomings that often discourage their teachers from using them for courses based on intense collaboration. It starts with the necessity to enrol staff and students into each module separately, which disregards the fact that a lot of students have similar courses and work together continuously. Because of the strong hierarchic model and the weak tools Moodle is not used for complex online project collaboration at all. Many partners use mash-up environments comprised of cutting-edge web services when it comes to innovations in creative collaboration. Aalborg's experiences confirm this, actually their modified Moodle version primarily allows one-way communication from teachers to students, which is why their students as well create their individual collaboration environments. However, for Isparta advanced Moodle features are at the core of their online teaching and the integration of the open source online meeting environment Big Blue Button within Moodle makes it a very powerful learning platform to them.

Ljubljana mentioned that university courses take place in uninspiring, formal settings. This applies to both physical and online spaces. They intend to create more playful environments that afford ideation and informal conversation, so the platform would match the open and constructivist-oriented approaches to learning in creative disciplines. On a more general level, TAMK remarked dyslexia is not uncommon among visually oriented people you find in design disciplines, which is why any collaboration environment should have a strong visual orientation and careful design, including accessibility aspects when it comes to complex forms. This is not the case with the text-centred Moodle system.

Further, TAMK mentioned team size as a critical factor, influencing a lot of parameters with regard to online collaboration. Trying to have online discussions in a plenary with 20 or more people does not afford participation and exchange. Magdeburg added, that even a change from 6 to 8 team members can make a huge change in the communication habits. 


\subsubsection{Emotional cues}

Emotional cues as such were not mentioned by the partners. However, closest to this is the practice to give space to colloquial conversation and activities in online collaboration as described by some partners. In the Mediaculture 2020 (Thayne and Cooper 2014) project, Lincoln deliberately set up a Facebook "coffee lounge" for informal discussions. In Magdeburg, online meetings often start with opening conversations and warm-up exercises to build up mutual empathy.

\subsubsection{Interoperability with different devices}

Creative online collaboration appears to happen on computers in the first place. There was no indication in the good practice research that other devices play a substantial role. More important seems the flexibility in content delivery. In the special case of online collaboration with African countries, the experience of Kassel is that the availability of computer equipment is a lesser problem than the unstable availability of electricity and internet connectivity. This is why in such cases the basic content should be restricted to downloadable text and image resources. Similar problems were mentioned by Lapland when they offered a master course on marketing to international students. For a number of students, their home universities in developing countries restricted the use of the internet access, so the students had to work from home or public internet cafés with weak connections. The solution was that local eLearning services downloaded and distributed the material to the students. Potsdam learned from international participants in their MOOC The Future of Storytelling that YouTube is banned in certain countries and that participants from Africa had to buy their downloadvolume at the beginning of a month and would need to know in advance if downloadintensive content will be exchanged in a course.

\subsubsection{Platform support}

The partner universities all have technical support for their respective online learning management systems (mainly Moodle). However, there seems to be a "digital divide" between faculty members. Some use the plethora of tools offered with confidence, others simply use the system to post lecture slides. There is little exchange on this within the faculties. In Magdeburg, training for teaching with Moodle is available, at Kassel the support is restricted purely to technical issues.

\subsection{Discussion of the results}

Across the different analysis categories, a picture of prevailing trends in creative online collaboration within the consortium emerged. These will serve as research hypotheses to shape the further research agenda within the OnCreate project.

1) Innovative collaboration concepts use mash-ups of cutting-edge services

The number of mentions of cloud storage services and apps was considerable, notably Google Drive and Google Apps. The same holds true for Facebook as a place for informal discussions and virtual socializing. It appeared that online 
collaboration settings are usually built around these basic tools and are enhanced with specialized tools such as online pinboards or dedicated project management services.

2) Existing learning management systems fail to support real creative collaboration

Related to the aforementioned observation, existing comprehensive eLearning solutions often lack acceptance for a variety of reasons. A strong disadvantage is the hierarchic role model, where only the teacher can modify the course environment, along with shortcomings of the integrated set of tools.

3) The classic teacher-student role model develops towards an open peer culture

All partners mentioned efforts to empower the students to reflect and control their own learning progress through various methods of self-assessment and peer collaboration. The focus of the teacher changes towards facilitating the reflected research and application of knowledge to build up competencies.

4) Basic connectivity and equipment issues mainly apply to highly intercultural settings While online collaboration was in its infancy, connectivity issues were a prevailing topic that seriously impaired communication or made it impossible. During this study such issues were only mentioned when acting in intercultural contexts beyond Europe. However, as the number of intercultural courses and projects can be expected to increase, these issues still need to be considered.

5) The creative process is not perceived as seriously limited in an online setting

This is a wide interpretation of the findings of the study, but derived from the fact that the challenges of unpredictable results and unique tasks where not mentioned as causing particular problems in an online context. There may be two complementary explanations for that. The first is that unpredictability and uniqueness in most projects relate to the actual implementation, but not to the general form of the desired result. This would mean that the creation of generic online spaces for certain groups of creative process is possible. The second explanation is that the adaption of tools happens mostly on the level of methods and usage conventions developed in a constant process of social ideation and negotiation. Very similar to a physical blackboard, also the online tools used by the OnCreate consortium could largely be insensitive to the actual method and content they are used for.

\section{Course implementation}

The OnCreate project implemented a number of pilot courses in order to explore new forms of creative online collaboration. These seek to implement in practice the findings of the research part of the OnCreate project. The purpose of introducing one particular course here is giving a further illustrative example of how the chosen categories for analysing the creative process and its contextual factors online translate to a practical context.

\subsection{Branding design course}

The Branding Design Course is a collaboration between four universities from the OnCreate network, namely Magdeburg, Tampere UoAS, Lincoln and Potsdam. The objective of the course is to learn designing logos and writing elementary style guides for corporate design. Four units each address particular challenges creative online collaboration (Table 4). 
Table 4 Course outline of OnCreate branding design course

\begin{tabular}{ll}
\hline Unit & Creative collaboration challenges \\
\hline $\begin{array}{l}\text { Reading and responding } \\
\text { to a design brief }\end{array}$ & $\begin{array}{c}\text {-Research on brand values, competition, inspiration and other } \\
\text { contextual requirements } \\
\text {-First rough ideation } \\
\text { Mood creation }\end{array}$ \\
$\begin{array}{l}\text { Logo creation } \\
\text { Styleguide creation }\end{array}$ & -Iterative sketching, selection, refinement and finalization \\
& -Development of a styleguide document \\
\hline
\end{tabular}

The course took place in the form of two 1-day workshops followed by 2 weeks of online collaboration each. Each workshop introduced 2 units with practical exercises on the design process, while iterations and refinements were done in smaller groups in the following online phase. The first workshop took place on site in Magdeburg, but integrated further online participants via a permanent Google Hangout projection on one wall of the room, while a second projection was used to display various collaboration tools that were chosen according to the task at hand.

Comparing the specific challenges of the course with the characteristics of creative collaboration (Table 2) we can assert that the mutual shared understanding was created especially by the fact that the groups had to do joint research based upon a project briefing. The team members published their findings on an online pinboard service, namely Realtimeboard. Further, the moodboard technique worked well to communicate a common frame to base design decisions on. Moodboards are collections of visual artifacts that approximate possible logo ideas in shape, colour and tone. In the offline world, moodboards usually have a scrapbook-look and are collected using magazine copies and glue \& scissors. For the online collaboration in the course the moodboards have been realized mostly as collections on pinterest.com. When it comes to unique tasks one can say that a brand design and more specifically a logo design has a quite clear scope when it comes to the form of the end product, compared e.g. to product design. So the tasks are in that sense unique that each logo briefing is very individual and there are countless possibilities to solve the problem. However, the mere steps of the design process are pretty clear. While those processes are not standardized in online collaborative environments yet, it can be assumed they could be in the near future. The main challenge is to find the right tools to exchange and discuss drafts. Students shared source files on a cloud storage service for co-creation, PDF via cloud storage or mail for commenting and some feedback was gathered using Adobe Behance, a sharing platform for digital artwork that has a "work-in-progress" feature that offers public comments. Joint decision-making appeared to be rather easy in the course, as it was always clear what was to be decided (i.e. choosing a design to iterate from an array of drafts) and the group size was small with 3 members per group. Also, given the nature of a compact course, the students settled quickly for a final draft to iterate and abandoned alternatives early to keep the workload for the course within reasonable boundaries. The teams work fully as peers, there was no hierarchy. Quite remarkably, even students with a background in design discussed openly and an equal level with students from other disciplines. They did not show any patronizing behaviour or impatience, which could have been expected given the differences in design 
experiences within the group. The design results showed a good variety. In the sense of the creative output they were unpredictable, yet of course they all were logos, following well-defined rules and design processes.

In contrast to more open creation processes following for example the design thinking paradigm (Lugmayr et al. 2013) the strongly predetermined formal nature of the creative output in visual design alleviates the problem of implementing very flexible processes. Moreover, flexibility can also be reached by using tools offering versatile uses. Online pinboards, for example, can be used to cluster research results as well as to visualize a moodboard or a storyboard. The methods are implemented via competencies inherent to the team member, not the software.

The course implemented some specific measures to facilitate creative collaboration. First, we used an authentic challenge and briefing for the course assignment. For the first run of the courses, we decided to ask for a logo for the OnCreate project. The coordinator of the OnCreate project was available for an interview based on the briefing, so the customer needs were not just made up and granted a sufficient depth of research findings to inspire well-thought design. The role of the teachers in this course was comparably conservative. They prepared the course self-learning material in a joint effort between four universities (i.e. Potsdam, TAMK, Lincoln and Magdeburg), gave introductory online lectures, facilitated some of the methods, especially collaborative design research and moodboarding and gave feedback in multiple iterations when the students started the visual design. So while a peer culture was prevailing among the students, the teachers were not part of it. Access to facilitation was constantly available through an asynchronous forum and Google Hangout chat. A part of the online collaboration happened throughout an entire day using a permanent Google Hangout session, Realtimeboard and Pinterest, ${ }^{5}$ which introduced an exciting multi-screen collaboration environment. The strongly video-focused online meeting service Google Hangout seemed to convey a good feeling of presence and immediacy that felt appropriate to the nature of the creative process.

There were notable observations regarding the use of online tools in the course in the project: First, the use of the online pinboard service Realtimeboard yielded a small but exciting example of adaptation of collaboration methods from the physical world to an online setting: The teams were collecting and sorting research results and requirements on virtual post-it notes, which works as a direct analogy to the method on a physical board. However, when they were discussing the research findings and requirements with the OnCreate project leader as stakeholder of the branding development process, they started to modify the size of post-it notes proportionally to its importance. This is an example of an implicitly emerging method triggered by the affordances of the software platform used.

The second observation was the comparison between physical and analogue methods of moodboarding. A moodboard is a collection of shapes, colours and motives that communicates an idea of the visual language to be developed for the branding design. Typically, a physical moodboard is compiled using images from used papers and magazines with glue and scissors. One group in the course created such a conventional moodboard, while the others used pinterest.com to collect images from the web. In comparison, the web version showed a larger variety in images, which is

\footnotetext{
${ }^{5}$ http://www.pinterest.com
} 
obvious as the students could use the whole web as a source for images, while the layout consisted of the typical pinterest.com vertical columns. The advantage of physical moodboards was the free placement of items, which allows to add a second layer of meaning by grouping and emphasizing. Also, there was an curious downside on drawing on web content for moodboarding: Usually moodboarding means looking for images that convey something, that is similar to a core creative idea. On the web, the students sometimes tended to find exactly what they were looking for, e.g. exactly the logo style imagined. If this is not reflected by the participants, it might actually curb creativity, as the found design item might already be satisfactory.

\section{Discussion of the draft framework}

The draft framework by and large provided a viable categorization for the research results of the good practice research in the OnCreate project. However, certain categories showed an excellent fit, while others seemed not to fit any core aspects of creative online collaboration in the practice of the universities involved.

The creative challenge of mutual exchange of viewpoints and knowledge seems to be a core concern to teachers within the OnCreate consortium. A lot of good practice examples addressed this and with convincing solutions. Although not dwelled on, joint decision-making appears also to be relevant to online collaboration curricula. And flat hierarchies both within student teams and between students and teachers fit show a general trend of establishing a peer culture in learning within the consortium. Thus it is sensible to use the three aforementioned categories for future iterations of the framework.

The challenges of unique tasks and unpredictable results were not as much recognized as posing problems in practice. It might be worthwhile to change the viewing angle here to the main requirement that could be expected from a theoretical point of view, which is the flexibility of creative processes. An important parameter might be the degree of pre-determination of the form of the final creative product, as discussed earlier. Also, it might very well be that these two challenges are agnostic towards whether the process is physical, digital, or any mix between both.

Among the contextual factors for online creative learning spaces, we see that the alignment between the model of learning and the communication model of the learning platform is a prime issue. It finds its expression in a clear tendency to test new web tools in innovative teaching courses instead of relying on the respective learning management platforms deployed at the universities. In one particular case, this even led to the development of a bespoke solution. It also appears that this alignment plays an important role in ensuring a good perceived user experience. A consequence might be to remove the generic factor user experience, as other factors describe already more targeted aspects of user experience. It would be advisable then to speak of the interaction model of the platform.

Under interoperability with different devices the expectations were to learn whether and how different mobile devices and operating systems used and supported. However, the issues concern target problems of intercultural collaboration with less developed regions and addressed basic problems of access to the platform. In terms of how different devices and learning contexts could be integrated into a creative online 
learning space the proper category to look at could rather be multimodal and contextspecific interaction, which in turn would be a sub-aspect of the alignment of the interaction model of the learning platform.

The continuous reflection of the collaboration process seems to be an established practice in online courses among the interviewed universities. The tendency to do this openly within the group and not only between the teacher and the student goes well along with the desire to create a peer learning culture in online collaboration courses. The factors access to facilitation and instructor feedback are strongly related to this, as they determine the role of the teacher. To be more specific, access to facilitation should be about how and through which channels the teacher communicates with the students, while instructor feedback should be about what kind of feedback is given.

Surprisingly, very few good practice examples explicitly addressed the lack of nonverbal communication in online collaboration settings and the need for substitution by providing emotional cues. There are a number of possible explanations for this, which provide interesting leads for future research. One hypothesis would be that online collaboration so far has been researched from a perspective that sets physical collaboration as a reference and researchers have been biased by their own experiences in physical collaboration, while online collaboration has been new to them. Yet now communication through online channels in voice, video, text and visuals is part of students' and teachers' daily lives. Thus, they developed genuine mental models about online based communication and know how to read and communicate subtext in new media, so they do not actually perceive the same communicative restriction that people deeply rooted in physical collaboration do experience. A second, more conservative hypothesis would be that in the major parts of the good practice examples the students were already familiar with at least some of the course participants and they worked together for at least a couple of weeks or even months. This is different from a situation where a team is built and collaborates purely online. Although the latter seems to be a prevailing assumption when researching on online collaboration, it is probably rather the exception than the rule.

\section{Conclusions and areas for future research}

The first research phase of the OnCreate project has revealed common trends in the implementation of creative online collaboration among the participating universities. Although these cannot be generalized from a scientific view point, they may serve as practical inspiration for universities seeking to promote the uptake of creative online collaboration in courses and projects:

1) Encourage coaching and peer-driven learning

2) Nourish a culture of open feedback and reflection

3) Encourage your staff and students to experiment with the latest online collaboration tools

4) Create authentic contexts for creative projects, e.g. by approaching industry partners

5) Evaluate and research creative online collaboration within its own context (and not from the perspective of a physical collaboration) 
Apart from a contribution to the practical discussion on creative online collaboration in education, the research results open a number of options for future research, which will help to iterate from the draft framework introduced in this paper towards a mature framework for the conception and evaluation of creative online collaboration processes. A first research strand is on the development of an open peer culture and the breakup of traditional hierarchies in the classroom. A fascinating question would be whether this development is only supported or rather driven by the availability of new collaboration technologies. As well, the changing role of the teacher in a world of abundant access to instructional material is a valid question and a shift towards coaching and facilitation might be a consequence of that. A second research strand lies at the intersection of education and user experience design and is about how we can enable teachers to recognize and describe interaction models of particular online tools and match them with their intended instructional design. Finally, the quality of the impact of online collaboration on the creative process and output has to be investigated in depths. Particularly this question will guide the next research phase in the OnCreate project, where it is intended to extend the good practice research towards protagonists in creative online collaboration outside the consortium in both education and industry.

Acknowledgments This work was co-funded by the European Union through the ERASMUS+ programme under grant agreement 2014-1-DE01-KA203-000706.

Open Access This article is distributed under the terms of the Creative Commons Attribution 4.0 International License (http://creativecommons.org/licenses/by/4.0/), which permits unrestricted use, distribution, and reproduction in any medium, provided you give appropriate credit to the original author(s) and the source, provide a link to the Creative Commons license, and indicate if changes were made.

\section{References}

Bagozzi, R. P., Davis, F. D., \& Warshaw, P. R. (1992). Development and test of a theory of technological learning and usage. Human Relations, 45(7), 659-686.

Brindley, J., Blaschke, L. M., \& Walti, C. (2009). Creating effective collaborative learning groups in an online environment. The International Review of Research in Open and Distributed Learning, 10(3). Retrieved from http://www.irrodl.org/index.php/irrodl/article/viewArticle/675

Brown, T. (2008). Design thinking. Harvard Business Review, 86(6), 84.

Burke, M., \& Settles, B. (2011). Plugged in to the community: Social motivators in online goal-setting groups. In Proceedings of the 5th International Conference on Communities and Technologies (pp. 1-10). ACM. Retrieved from http://dl.acm.org/citation.cfm?id=2103356

Buxton, B. (2010). Sketching user experiences: Getting the design right and the right design: Getting the design right and the right design. Morgan Kaufmann.

Davis, F. D., Bagozzi, R. P., \& Warshaw, P. R. (1989). User acceptance of computer technology: a comparison of two theoretical models. Management Science, 35(8), 982-1003.

Doorley, S., Witthoft, S., \& others. (2011). Make space: How to set the stage for creative collaboration. John Wiley \& Sons.

Dow, S., \& Settles, B. (2013). Modeling online creative collaborations. XRDS: Crossroads, the ACM Magazine for Students, 19(4), 21-25.

Estellés-Arolas, E., \& González-Ladrón-de-Guevara, F. (2012). Towards an integrated crowdsourcing definition. Journal of Information Science, 38(2), 189-200.

Godwin-Jones, R. (2003). Emerging technologies: Blog and Wikis: Environments for on-line collaboration. Virginia Comonwealth University.

Haythornthwaite, C. (2006). Facilitating collaboration in online learning. Journal of Asynchronous Learning Networks, 10(1), 7-24. 
Herrington, A., \& Herrington, J. (2006). Authentic learning environments in higher education. IGI Global. Herrington, J., \& Parker, J. (2013). Emerging technologies as cognitive tools for authentic learning. British Journal of Educational Technology, 44(4), 607-615.

Holmes, J. (2012). Education futures: University grads don't make the grade (Educations Futures). Woods Bagot. Retrieved from http://ctibi2012.wikispaces.com/file/detail/Business_Schools_EducationFutures.pdf

Hong, S. W. (2013). The affordance of online multiuser virtual environments for creative collaboration. University of California, Berkeley. Retrieved from http://escholarship.org/uc/item/91r0m1sx.pdf

Jäminki, S. (2008). Ohjaus-ja opiskeluprosessit samanaikaisessa ja eriaikaisessa verkkoympäristössä: etnografinen tutkimusmatka verkkotutkinnon maailmaan. Lapin yliopisto.

Johnson, D. W., \& Johnson, R. T. (1996). Cooperation and the use of technology. Handbook of Research for Educational Communications and Technology: A Project of the Association for Educational Communications and Technology, 1017-1044.

Kalay, Y. E. (2006). The impact of information technology on design methods, products and practices. Design Studies, 27(3), 357-380.

Kim, J., Cheng, J., \& Bernstein, M. S. (2014). Ensemble: Exploring complementary strengths of leaders and crowds in creative collaboration. In Proceedings of the 17th ACM conference on Computer supported cooperative work \& social computing (pp. 745-755). ACM. Retrieved from http://dl.acm.org/citation. cfm?id=2531638

Lahti, H., Seitamaa-Hakkarainen, P., \& Hakkarainen, K. (2004). Collaboration patterns in computer supported collaborative designing. Design Studies, 25(4), 351-371.

Leidner, D. E., \& Jarvenpaa, S. L. (1995). The use of information technology to enhance management school education: a theoretical view. MIS Quarterly, 265-291.

Lugmayr, A., Stockleben, B., Zou, Y., Anzenhofer, S., \& Jalonen, M. (2013). Applying 'Design Thinking' in the context of media management education. Multimedia Tools and Applications, 71(1), 119-157. doi:10. 1007/s11042-013-1361-8.

Okuogume, A., \& Jäminki, S. (2011). Experiences of integrating R\&D\&I in professional master degree programme: The case of competence-development-based education. Hämeenlinna: Presented at the Combi Conference.

Richardson, J. C., \& Swan, K. (2003). Examing social presence in online courses in relation to students' perceived learning and satisfaction. Retrieved from https://www.ideals.illinois.edu/handle/2142/18713

Romero, M., \& Barberà, E. (2014). Computer-based creative collaboration in online learning. In D. W. Chiu, M. Wang, E. Popescu, Q. Li, \& R. Lau (Eds.), New horizons in web based learning (Vol. 7697, pp. 330336). Springer Berlin Heidelberg. Retrieved from http://dx.doi.org/10.1007/978-3-662-43454-3_34

Runco, M. A., \& Jaeger, G. J. (2012). The standard definition of creativity. Creativity Research Journal, 24(1), 92-96.

Schoenfeld, A. H. (1999). The core, the canon, and the development of research skills: issues in the preparation of education researchers. Issues in Education Research: Problems and Possibilities, 166-202.

Schrage, M. (1995). No more teams!: Mastering the dynamics of creative collaboration. New York: Currency Doubleday.

Selim, H. M. (2007). Critical success factors for e-learning acceptance: confirmatory factor models. Computers \& Education, 49(2), 396-413.

Shea, P., Li, C. S., Swan, K., \& Pickett, A. (2005). Developing learning community in online asynchronous college courses: The role of teaching presence. Journal of Asynchronous Learning Networks, 9(4), 59-82.

Slavin, R. E. (1980). Cooperative learning. Review of Educational Research, 50(2), 315-342.

Swan, K., \& Shih, L. F. (2005). On the nature and development of social presence in online course discussions. Journal of Asynchronous Learning Networks, 9(3), 115-136.

Swan, K., Garrison, D. R., \& Richardson, J. (2009). A constructivist approach to online learning: The community of inquiry framework. information technology and constructivism in higher education: Progressive learning frameworks (pp. 43-57). Hershey: IGI Global.

Swan, K., Shea, P., Frederickson, E., Pickett, A., Pelz, W., \& Maher, G. (2000). Building knowledge-building communities: Consistency, contact and community in the virtual classroom. Albany: SUNY Learning Network.

Thayne, M., \& Cooper, G. (2014). Collaborative pedagogy and digital scholarship: a case study of 'media culture 2020. Media Education Research Journal (MERJ), 5(1), 46-59.

Thayne, M., Stockleben, B., Jäminki, S., Haukijärvi, I., Mavengere, N., Demirbilek, M., \& Ruohonen, M. (2015). OnCreate and the virtual teammate: an analysis of online creative processes and remote collaboration. Retrieved from http://eprints.lincoln.ac.uk/17816

Vygotsky, L. (1978). Mind in society: The development of higher psychological processes. Cole, M., et Al., LS Vygotsky. Harvard University Press, Cambridge. 